\title{
Pulsar Wind Nebulae and Their Supernovae
}

\author{
Roger A. Chevalier \\ Department of Astronomy, University of Virginia, P.O. Box 3818, \\ Charlottesville, VA 22903, USA
}

\begin{abstract}
Young supernova remnants that contain pulsar wind nebulae provide diagnostics for both the inner part of the supernova and the interaction with the surrounding medium, providing an opportunity to relate these objects to supernova types. Among observed young nebulae, there is evidence for a range of supernova types, including Type IIP (Crab Nebula and SN 1054) and Type IIb/IIn/IIL (G292.0+1.8).
\end{abstract}

\section{Introduction}

Recent X-ray satellites ( $A S C A$, Chandra, and XMM-Newton) have substantially increased the number of observed young pulsar wind nebulae (PWNe), as well as adding to our knowledge of the nebulae and their surrounding supernova remnants (SNRs). Young PWNe are expected to be interacting with freely expanding ejecta in the interior of SNRs, providing a probe of the inner parts of the supernova. The surrounding SNR is typically interacting with circumstellar mass loss from the progenitor star. The combination of this information can be related to our expectations for core-collapse supernovae and their surroundings. Here, I examine the properties of eight young PWNe in this context.

\section{Supernovae and Their Surroundings}

Recent observations of core-collapse supernovae at X-ray and radio wavelengths have yielded a fairly complete picture of the circumstellar surroundings of the various supernova types (Chevalier 2003). Although the numbers are still small, Type IIP (plateau) supernovae have been found to be relatively weak X-ray and radio sources, with a mass loss density corresponding to a mass loss rate $\dot{M} \approx 10^{-6} \mathrm{M}_{\odot} \mathrm{yr}^{-1}$ for a wind velocity of $v_{w}=10 \mathrm{~km} \mathrm{~s}^{-1}$; the light curves of these supernovae indicate red supergiant progenitors. The low mass loss is consistent with the fact that the supernova properties indicate that most of the stellar $\mathrm{H}$ envelope is present at the time of the explosion. A consequence of this is that the inner heavy element core is decelerated by the envelope and mixes with it, giving rise to low-velocity $\mathrm{H}$ in the supernova. There is information on the progenitor stars of 2 Type IIP supernovae, leading to upper mass limits of the progenitors of $12 \mathrm{M}_{\odot}$ and $15 \mathrm{M}_{\odot}$ (Smartt et al. 2003). Thus, SNe IIP appear to be near the lower mass limit for core-collapse supernovae.

$\mathrm{SN} 1987 \mathrm{~A}$ is another supernova that exploded with a massive $\mathrm{H}$ envelope that was able to decelerate the heavy element ejecta. However, the progenitor 
in this case was a blue supergiant with a mass $\sim 19 \mathrm{M}_{\odot}$. The immediate surrounding of the blue supergiant star was determined by the fast wind from that star but, further out, beyond the ring at $0.2 \mathrm{pc}$, there is evidence for a slow red supergiant wind (Crotts 2000).

Supernovae that show strong interaction with a dense red supergiant wind are of Types IIn (narrow line), IIL (linear), and IIb (evolve to Ib/c spectrum); these types are not mutually exclusive as they refer to different aspects of the supernova. In these cases, the presupernova mass loss can go up to $\dot{M} \approx 10^{-4}$ $10^{-3} \mathrm{M}_{\odot} \mathrm{yr}^{-1}$ for $v_{w}=10 \mathrm{~km} \mathrm{~s}^{-1}$. In $\mathrm{SNe}$ IIb and at least some IIn (e.g., Fransson et al. 2002), most of the $\mathrm{H}$ envelope is lost before the supernova. The result is that there is no slow $\mathrm{H}$ in the supernova; e.g., in the IIb $\mathrm{SN} 1993 \mathrm{~J}, \mathrm{H}$ was in the range $8500-10,000 \mathrm{~km} \mathrm{~s}^{-1}$ (Houck \& Fransson 1996).

The dense wind from a red supergiant progenitor star can extend out some distance around the progenitor. The wind comes into pressure equilibrium with the surrounding medium at a radius (Chevalier \& Emmering 1989)

$$
r_{s h}=5.0\left(\frac{\dot{M}}{5 \times 10^{-6} \mathrm{M}_{\odot} \mathrm{yr}^{-1}}\right)^{1 / 2}\left(\frac{v_{w}}{15 \mathrm{~km} \mathrm{~s}^{-1}}\right)^{1 / 2}\left(\frac{p_{i}}{10^{3} \mathrm{~cm}^{-3} \mathrm{~K}}\right)^{-1 / 2} \mathrm{pc}
$$

where $p_{i}$ is the external pressure. This expression assumes that the wind has been steady for at least a time $r_{s h} / v_{w}$, or $3.3 \times 10^{5}$ years for the reference values. The red supergiant wind may end in a shell, which may have been observed around SN 1987A (Chevalier \& Emmering 1989). Beyond the red supergiant wind, there may be a wind bubble created by the progenitor star when it was on the main sequence. Although the wind bubble in a low density uniform medium can be large, interstellar clouds could be relatively close to the progenitor.

The remaining types of core collapse supernovae are the Type Ib and Ic explosions, which are thought to have massive star progenitors that lost their $\mathrm{H}$ envelopes. In this case, no slow $\mathrm{H}$ is expected in the supernova, and the immediate surroundings of the supernova are determined by the low density, fast wind typical of a Wolf-Rayet $\operatorname{star}\left(\dot{M} \approx 10^{-5} \mathrm{M}_{\odot} \mathrm{yr}^{-1}\right.$ and $\left.v_{w} \approx 10^{3} \mathrm{~km} \mathrm{~s}^{-1}\right)$. The combination of stellar mass loss estimates with the fact that stars with an initial mass $\gtrsim 25 \mathrm{M}_{\odot}$ collapse to black holes suggests that Type Ib and Ic are the result of binary evolution (Heger et al. 2003). In this case, a substantial part of the $\mathrm{H}$ envelope mass loss is due to the companion star.

Dahlén \& Fransson (1999) have summarized recent work on supernova rates, finding the following intrinsic fractions of core-collapse supernova types: SN IIP (0.3), SN IIL (0.30), SN IIn (0.02), SN 1987A-like (0.15), SN Ib/c (0.23); the rate of $\mathrm{SN} 1987 \mathrm{~A}$-like events is especially uncertain. Unless there is a strong correlation of pulsars with supernova types, PWNe should appear in the young remnants of a variety of supernova types.

\section{Young Pulsar Wind Nebulae}

Table 1 lists eight young PWNe that are plausibly interacting with freely expanding ejecta from the supernova (see Chevalier 2004 for references to these objects). Tentative supernova identifications have been given in four cases, but only the identification of the Crab Nebula with SN 1054 can be considered secure. In two other cases (0540-69.3 and G292.0+1.8), there is an age estimate 
from the expansion of optical filaments. $R_{s n r}$ is the radius of the surrounding supernova remnant except where no surrounding remnant is observed, in which case the radius of the PWN is given (in parentheses). $V_{s n r}$ is the average velocity of the remnant obtained by dividing $R_{s n r}$ by the age.

Table 1. Properties of young pulsar nebulae.

\begin{tabular}{cccccc}
\hline $\begin{array}{c}\text { Supernova } \\
\text { Remnant }\end{array}$ & $\begin{array}{c}P / 2 P \\
(\mathrm{yr})\end{array}$ & $\begin{array}{c}\text { Age } \\
(\mathrm{yr})\end{array}$ & SN & $\begin{array}{c}R_{\text {snr }} \\
(\mathrm{pc})\end{array}$ & $\begin{array}{c}V_{\text {snr }} \\
\mathrm{km} \mathrm{s}^{-1}\end{array}$ \\
\hline 0540-69.3 & 1660 & 760 & & 9 & 11,600 \\
3C 58 (G130.7+3.1) & 5390 & 821 & 1181 & $(3.3)$ & $(3,900)$ \\
Crab (G184.6-5.8) & 1240 & 948 & 1054 & $(2)$ & $(2,100)$ \\
Kes 75 (G29.7-0.3) & 723 & & & 9.7 & 13,000 \\
G11.2-0.3 & 24000 & 1616 & 386 & 3.3 & 2,000 \\
G292.0+1.8 & 2890 & $\lesssim 1700$ & & 5.8 & 3,200 \\
MSH 15-52 (G320.4-1.2) & 1700 & 1817 & 185 & 20 & 10,800 \\
G54.1+0.3 & 2890 & & & $(1.3)$ & $(440)$ \\
\hline
\end{tabular}

There are several arguments for associating the Crab Nebula and SN 1054 with a SN IIP. The presence of $\mathrm{H}$ in the ejecta suggests that the core material was decelerated by the $\mathrm{H}$ envelope, as expected in a SN IIP. The abundances in the Crab imply a progenitor mass of $\sim 9 \mathrm{M}_{\odot}$ (Nomoto 1985), which is compatible with SN IIP. Finally, the lack of a shell around the Crab implies that the supernova is interacting with a low density surrounding medium. The low radio and X-ray luminosities of SN IIP suggest that they have a relatively low density wind, which may not extend far from the progenitor (Eq. [1]). The supernova may have swept through the wind and is now in a low density bubble. This scenario for SN 1054 was outlined in Chevalier (1977), but the absence of any observable interaction around the Crab has frustrated attempts at verification.

There are no ejecta observations in $3 \mathrm{C} 58$, but the presence of slow moving, $\mathrm{N}$-rich flocculi (Fesen, Kirshner \& Becker 1988) is suggestive of a clumpy red supergiant wind surrounding the progenitor. A SN IIP explosion is thus a possibility, with the lack of a surrounding shell being due to a relatively small amount of mass loss. For G54.1+0.3, the lack of a surrounding shell suggests that it is not a SN IIb/IIn/IIL, but there is not enough information on the object to be more definite.

The pulsar and PWN in 0540-69.3 have many similarities to the Crab and its pulsar, but there is a shell present in this case. The presence of $\mathrm{H}$ in the shell swept up by the PWN has been controversial (Kirshner et al. 1989); if it is present, a SN IIP explosion is indicated. The progenitor mass would be somewhat larger that for SN 1054 because of the enhanced $\mathrm{O}$ emission from the ejecta (initial mass $\gtrsim 13 \mathrm{M}_{\odot}$ ). The large size of the shell and the high average velocity imply that the supernova encountered little circumstellar matter before running into denser, inhomogeneous surrounding gas; i.e., the supernova shock traversed a stellar wind bubble. The large sizes and velocities observed in MSH 15-52 and Kes 75 suggest a similar situation in these objects, implying that they did not result from Type IIb/IIn/IIL supernovae. On the basis of the high 
energy/mass ratio deduced for MSH 15-52, Gaensler et al. (1999) suggested a $\mathrm{SN}$ Ib origin; the finding of $\mathrm{H}$-free ejecta would help to confirm this designation.

The remaining two objects, G11.2-0.3 and G292.0+1.8, have remnants with both a small radius and low velocity, suggesting strong circumstellar interaction and a SN IIb/IIn/IIL origin. The red supergiant wind from the progenitor star can extend to the observed radii, so this is the likely ambient medium. Strong mass loss is consistent with the absence of Balmer lines in the spectrum of G292.0+1.8 (Goss et al. 1979), implying that most of the $\mathrm{H}$ envelope had been lost before the supernova.

These considerations show that supernova remnants around young $\mathrm{PWNe}$ are likely to be strongly influenced by the presupernova mass loss. Future observations of ejecta abundances and additional observations of the circumstellar interaction should allow clearer associations with supernova types.

Acknowledgments. This work was supported in part by NASA grant NAG5-13272.

\section{References}

Chevalier, R. A. 1977, in Supernovae, ed. D. N. Schramm (Dordrecht: Reidel), p. 53

- 2003, in From Twilight to Highlight: The Physics of Supernovae, eds. W. Hillebrandt, \& B. Leibundgut (Berlin: Springer), p. 299

Chevalier, R. A. 2004, Adv. Sp. Res., 33, 456

Chevalier, R. A., \& Emmering, R. T. 1989, ApJ, 342, L75

Crotts, A. P. S. 2000, in ASP Conf. Ser., Vol. 199, Asymmetrical Planetary Nebulae II, eds. J. H. Kastner, N. Soker, \& S. Rappaport, (San Francisco: ASP), p. 445

Dahlén, T., \& Fransson, C. 1999, A\&A, 350, 349

Fesen, R. A., Kirshner, R. P., \& Becker, R. H. 1988, in Supernova Remnants and the Interstellar Medium, eds. R. S. Roger, \& T. L. Landecker, (Cambridge: CUP), p. 55

Fransson, C., et al. 2002, ApJ, 572, 350

Gaensler, B. M., Brazier, K. T. S., Manchester, R. N., Johnston, S., \& Green, A. J. 1999, MNRAS, 305, 724

Goss, W. M., Shaver, P. A., Zealey, W. J., Murdin, P., \& Clark, D. H. 1979, MNRAS, 188, 357

Heger, A., Fryer, C. L., Woosley, S. E., Langer, N., \& Hartmann, D. H. 2003, ApJ, 591, 288

Houck, J. C., \& Fransson, C. 1996, ApJ, 456, 811

Kirshner, R. P., Morse, J. A., Winkler, P. F., \& Blair, W. P. 1989, ApJ, 342, 260

Nomoto, K. 1985, in The Crab Nebula and Related Supernova Remnants, eds. M. C. Kafatos, \& R. C. B. Henry, (Cambridge: CUP), p. 97

Smartt, S. J., Maund, J. R., Gilmore, G. F., Tout, C. A., Kilkenny, D., \& Benetti, S. 2003, MNRAS, 343, 735 